Article

\title{
Differences in the Gaze Behaviours of Pedestrians Navigating between Regular and Irregular Road Patterns
}

\author{
Bing Liu ${ }^{1,2}$, Weihua Dong ${ }^{1, *}$, Zhicheng Zhan ${ }^{1}$, Shengkai Wang ${ }^{1}\left(\mathbb{D}\right.$ and Liqiu Meng ${ }^{2}(\mathbb{D}$ \\ 1 Beijing Key Laboratory for Remote Sensing of Environment and Digital Cities, Research Center of Geospatial \\ Cognition and Visual Analytics and Faculty of Geographical Science, Beijing Normal University, \\ Beijing 100875, China; liubing_geo@mail.bnu.edu.cn (B.L.); zhanzhicheng@mail.bnu.edu.cn (Z.Z.); \\ wangsk@mail.bnu.edu.cn (S.W.) \\ 2 Chair of Cartography, Technical University of Munich, 80333 Munich, Germany; liqiu.meng@tum.de \\ * Correspondence: dongweihua@bnu.edu.cn; Tel.: +86-10-5880-9246
}

Received: 11 December 2019; Accepted: 13 January 2020; Published: 15 January 2020

\begin{abstract}
While a road pattern influences wayfinding and navigation, its influence on the gaze behaviours of navigating pedestrians is not well documented. In this study, we compared gaze behaviour differences between regular and irregular road patterns using eye-tracking technology. Twenty-one participants performed orientation (ORI) and shortest route selection (SRS) tasks with both road patterns. We used accuracy of answers and response time to estimate overall performance and time to first fixation duration, average fixation duration, fixation count and fixation duration to estimate gaze behaviour. The results showed that participants performed better with better accuracy of answers using irregular road patterns. For both tasks and both road patterns, the Label areas of interest (AOIs) (including shops and signs) received quicker or greater attention. The road patterns influenced gaze behaviour for both Road AOIs and Label AOIs but exhibited a greater influence on Road AOIs in both tasks. In summary, for orientation and route selection, users are more likely to rely on labels, and roads with irregular patterns are important. These findings may serve as the anchor point for determining how people's gaze behaviours differ depending on road pattern and indicate that labels and unique road patterns should be highlighted for better wayfinding and navigation.
\end{abstract}

Keywords: road pattern; orientation; shortest route selection; eye tracking

\section{Introduction}

Road patterns provide fundamental information for mobile activities, such as wayfinding, route planning and automatic navigation; however, they are also complicated. Road patterns vary in heterogeneity, connectivity, accessibility, interconnectivity [1], etc. [2,3]. These sophisticated systems not only impact the performance of transportation systems [4] and land use [5] but also strongly influence people's behaviours [6-10]. Many researchers have focused on the geometric attributes of road patterns and navigation solutions based on shortest time/distance routes [11,12] or other objective conditions [13]. However, people do not act solely based on geometric attributes. For example, drivers frequently do not take the shortest time route, and pedestrians have even more freedom in their movement choice [14]. When selecting a route, people tend to choose straight roads near the origin [15]. Pedestrians' perceptions of travel time are influenced by the network structure [2,16], which may influence their route selection. Therefore, it is necessary to investigate how people perceive and interact with road patterns and which information is important in this process.

Researchers have long been interested in the influence of road patterns on behaviour, such as driving performance, traffic safety [17] and route choice. Jacob et al. [18] and Green [19] found an 
increase in workload for drivers at smaller-radius and higher-deflection-angle curves in rural areas. Contrary to the common opinion that bends are dangerous for traffic, Haynes et al. [20] found that from a district perspective, straighter roads result in more crashes, and fatal road crashes are negatively related to the angle of roads. Zhang et al. [21] analysed the associations between non-motorist (i.e., pedestrians and cyclists)-involved crashes and the road network structure in Alameda County, California, and found that more intersections between pairs of roads tend to be safer for pedestrians. This study indicated that planners could block cut-through paths to improve traffic safety for pedestrians. However, the inconvenience caused by more intersections might prevent pedestrians from using them, which highlights the importance of considering people's feelings. In addition, people's route choices are also affected by road patterns, as shown by studies based on global positioning system (GPS) commute routes [2,16], actual walking conditions [22-25] and experimental conditions [26,27]. Research by Hochmair and Karlsson [28] on strategy preference in route selection indicates that different cognition processes occur between map-based and view-based navigations. For example, map-based route choice tends to include longer initial straight segments, while view-based users prefer short segments. Both Parthasarathi, Levinson, and Hochmair [2] and D'Acci [25] found that the road pattern influences pedestrians' time perception, with participants in the latter study preferring curvy roads.

Researchers have attempted to explain these behavioural differences. Behaviour-based studies in the physical world indicate that these differences may be related to the influence of road patterns on the judgement of geospatial metrics. Byrne [29] found that participants tend to overestimate the lengths of short routes and routes with major bends but not straight routes. Meanwhile, the estimations of intersection angles tend to be approximately $90^{\circ}$ regardless of the actual angle $\left(60-70^{\circ}\right.$ or $110-120^{\circ}$ ) in their residential neighbourhood. R. Montello [30] asked sixty pedestrians in three testing areas (one orthogonal and two oblique to the local grid pattern) to point to several nonvisible local targets or the main route direction. He compared the pointing accuracies and response times in these grids, and the results showed that the participants pointed more accurately with orthogonal streets than oblique streets. Due to complicated conditions in the real world, recent studies directly examine cognition processes with different road patterns in highly controlled laboratory conditions. For example, Liu et al. [31] reported research on cognition with different road patterns based on an fMRI (functional magnetic resonance imaging) experiment. They observed greater activation in cognition- and eye-movement-related brain areas in an orientation task with an irregular road pattern (compared with a regular road pattern), which indicates that orientating with an irregular road pattern is more difficult. These studies show that people's behaviours and cognition can be influenced by road patterns. However, as the road patterns in such studies are integrally regarded, it is difficult to clarify the aspects of the road pattern responsible for the differences. In this study, we separately analysed road and labels to distinguish the influencing component.

Eye-tracking technology, which is based on the eye-mind assumption [32], is commonly used to determine how people process information [32-36]. Because walking on roads requires considerable visual information and many attention switches, eye-tracking technology is applicable for navigation [37] and road-related [34,38] research. Hepperle and von Stülpnagel [39] compared gaze behaviour during intentional and incidental route learning and retrieval and found that the main difference pertained to the objects that the participants did not view. Liao et al. [40] used eye movement data to infer pedestrians' navigation tasks from five possible tasks and obtained a total classification accuracy of $67 \%$. Fotios et al. [38] investigated the proper illumination design for pedestrians after dark by analysing where people fixated their attention and concluded the importance of providing sufficient illumination for other people and paths. However, this study was conducted during a walking period and did not specify certain tasks. Kitazawa and Fujiyama [41] analysed participants' eye tracking data while walking and found that pedestrians usually focus on the scene directly in front of them and that the information-processing space resembled a cone. Trefzger et al. [42] indicated that pedestrians and cyclists paid the most attention to the path during navigation. Giannopoulos, Kiefer, and Raubal [43] also applied eye-tracking technology to assist in the navigation of pedestrians and installed their 
GazeNav app on a smartphone. This app makes the smartphone vibrate if the user looks at the correct street. These studies indicate that eye movement data are valuable in road-related research.

In this study, we aimed to identify pedestrian gaze differences between regular road patterns and irregular road patterns for navigational tasks and identify the roles of label and road information in this process. We categorized road patterns into irregular and regular patterns, asked participants to perform orientation (ORI) and shortest route selection (SRS) tasks using screenshots of the street view in both types of road patterns, and recorded the participants' eye movements during the tasks. By analysing eye movements over road and label information, we investigated the information that is important for navigational tasks and the differences among distinct road patterns. The results provide insights into improved road designs.

The second section of this paper introduces the experimental methods, including the experimental design overview, participants, apparatus, materials, procedure and data analysis methods. The third and fourth sections report the results and discuss the results, respectively. The fifth section concludes this study and proposes future work.

\section{Materials and Methods}

\subsection{Experimental Design}

In this study, roads were classified into two categories according to their patterns. Roads with orthogonal intersections and straight segments were regarded as regular road patterns, and those with non-orthogonal intersections or curved segments as irregular patterns [39,44]. We applied a within-participants design, where participants were instructed to perform ORI and SRS tasks in both patterns. These tasks were chosen because they presented a sense of orientation and distance. As part of a large-scope project of cognition research, this eye-tracking-based study shares a similar experimental design with previous work reported in [31]. However, the results of this previous study only confirmed the influence of road patterns and could not explain which part of the road pattern was attributed to in the influence.

The experiment was performed on two consecutive days. On Day 1, participants were instructed to become familiar with two areas-an area with a regular road pattern and an area with an irregular road pattern via street view maps. On Day 2, the participants accomplished a set of ORI and SRS tasks based on the previously learned areas, while their eye movements were recorded. Although this study was based on a newly learned road network and participants were perhaps not able to create a complete cognitive map, this map was not necessary to perform a successful geospatial task [45]. Before the experiment, we conducted a pre-test in which five university students from Beijing Normal University (BNU) participated to verify the materials and procedure in the experiment.

\subsection{Participants}

Twenty-three students who were recruited via online ads from universities in Beijing participated in the experiment. Two of the students did not complete the experiment due to reported difficulties in learning or unease during the experiment. The remaining 21 participants (mean age $=22.4, \mathrm{SD}=2.3$; 7 males and 17 females; 7 with geography-related background) completed the experiment. With respect to the preferred reference system, six participants reported a preference for an allocentric reference system (i.e., using east/west/north/south) for wayfinding, ten preferred egocentric (right/left) and five reported no preferences and used both.

All of the participants had normal or corrected-normal eyesight, and none of the participants reported a history of mental illnesses. Seven records for the ORI task and three records for the SRS task were excluded because the sample rates (i.e., the percentage of eye movement data that is recorded) were below $70 \%$. Therefore, 14 eye movement datasets (five from males) in the ORI task and 18 eye movement datasets (five from males) in the SRS task were analysed. Each participant received 160 RMB as compensation. 


\subsection{Apparatus}

The experiment was conducted in a quiet lab in BNU. We used the Tobii T120 eye tracker (Tobii AB, Stockholm, Sweden; www.tobii.com) with the matching Tobii Studio 3.2.1 software in this study to record the participants' eye movements and export the data. The hardware included a Tobii eye tracker with a $60 \mathrm{~Hz}$ sampling rate and a 17-inch thin-film-transistor (TFT) monitor with a $1280 \times 1024$ pixel screen resolution. The recording accuracy of the eye tracker was $0.5^{\circ}$ with $0.2^{\circ}$ spatial resolution. The allowed head movement range was $0.2^{\circ}$. The tracking distance ranged from 50 to $80 \mathrm{~cm}$, and in this experiment, the distance between the participant and the monitor was approximately $60 \mathrm{~cm}$.

\subsection{Materials}

In this study, we used street views from Google Maps as the experimental material to eliminate the influence of other pedestrians [38], weather and traffic conditions. We chose part of Stamford, Lincolnshire, the United Kingdom and part of Ashton-under-Lyne, Greater Manchester, the United Kingdom as the irregular and regular road patterns, respectively (Figure 1, these maps were only for design purposes and were not shown to participants). To eliminate memory effects, we chose these two study areas in the UK to ensure that the participants were not previously familiar with the areas. The stimuli should be understandable to the participants, as they are university students and have taken the English exam in the National College Entrance Examination. None of the five participants who were recruited during the pre-test reported learning difficulties that were attributed to the English environment.

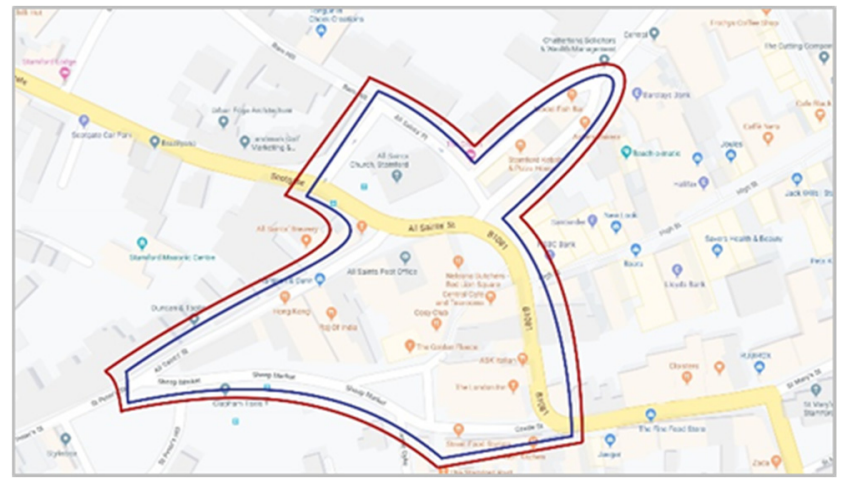

(a)

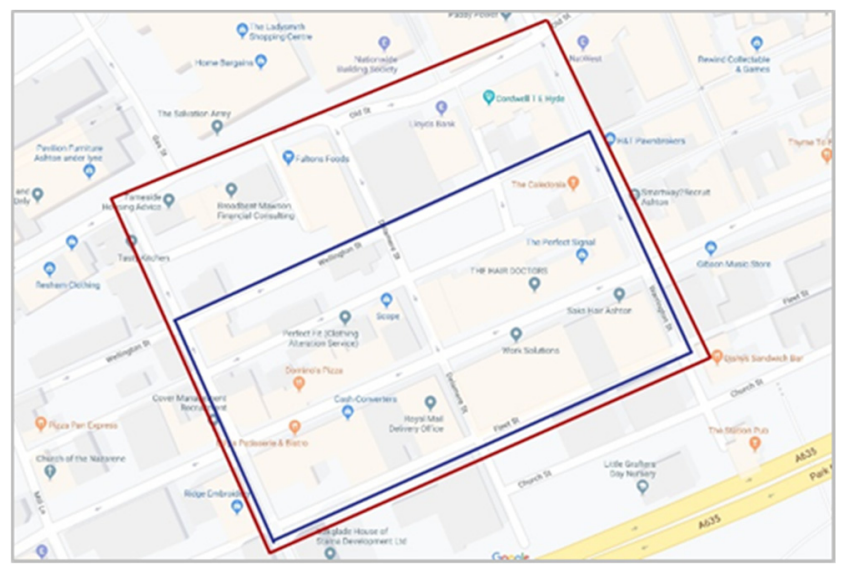

(b)

Figure 1. Experimental areas: (a) Stamford, Lincolnshire and (b) Ashton-under-Lyne, Greater Manchester. Red indicates the pre-test area and blue represents the experimental areas; participants were not provided with these maps. 
Both of the study areas consisted mainly of business areas in small towns, where the majority of buildings had two or three floors and various signs and labels. Thus, the street view of the areas did not show an excessive number of people. The initial experimental areas were within the red framework, as shown in Figure 1. After the pre-test, part of the regular experiment area was cut off as the participants indicated that this area generated a larger workload and they needed more time to learn compared with the irregular area. The final experimental areas are shown in the blue framework.

On Day 1, when the participants were asked to remember the experiment areas, the controlling panels (e.g., the small map window and information box used on Google Maps) were hidden using the Google Maps application programming interface (API, Google [46]).

Street view screenshots $(1024 \times 640)$ of these areas were used as materials for the ORI and SRS tasks on Day 2. All the street view screenshots were unique and used only once. In these screenshots, names of streets were also hidden, as there are usually no names painted on streets. We did not use dynamic or interactive materials in the ORI and SRS tasks because the interaction itself might influence the visual attention distribution, and controlling the delay of updating was difficult if we used prepared dynamic videos.

\subsection{Procedure}

Day 1 As the participants did not know the study areas in any form before the experiment, they were asked to become familiar with these areas on Day 1. First, the researchers introduced the experimental timetable, including a sample task. The participants provided a signed consent form and were told that they could quit during any phase of the experiment.

The participants were then guided to "walk" along the boundaries of the irregular road pattern in Google Street View. They were allowed to navigate in the area freely by mouse or keyboard and were required to remember this area. Once the participants reported what they had remembered in this area, they were shown 10-12 screenshots of the street view and asked to indicate whether the screenshots displayed the roads that they had learned within the previous $5 \mathrm{~s}$ (for each screenshot). The participants needed to achieve at least $90 \%$ accuracy to begin the same procedure for the regular road pattern; otherwise, they needed to repeat learning and testing for the irregular road pattern.

Day 2 The participants were first shown and explained the instructions without using eye tracking. They needed to perform both ORI and SRS example tasks to ensure that they had fully understood the instructions (as described in [31], which employed the Baidu Streetview (Baidu Map: https://map.baidu.com/) near BNU to ensure the participants were familiar with the environment).

The ORI and SRS tasks with eye tracking then began. A five-point calibration was used. During this task phase, no further instruction was provided unless the task section changed (i.e., from ORI to SRS). The tasks were presented in the order of ORI tasks in an irregular road pattern (irORI), ORI tasks in a regular pattern (rORI), SRS tasks in an irregular road pattern (irSRS) and SRS tasks in a regular pattern (rSRS). Each part consisted of 10 subtasks. After the rORI part, the participants were allowed to rest their eyes. In each subtask, first, a white cross was presented in the middle of a black page for $1 \mathrm{~s}$ (as Figure 2 shows). Second, a screenshot of the destination (Figure 3a) was displayed for $6 \mathrm{~s}$ and, last, a screenshot of the current position was shown (Figure 3b for ORI and Figure $3 \mathrm{c}$ for SRS, tasks described with the figures). At this point, the participants were allowed to make a choice using their keyboard without time limitations. To prevent the participants from randomly guessing, they were allowed to press the space bar to skip a subtask if they could not recall the roads. Once they made their choice, the next subtask began with the black page with a white cross. 


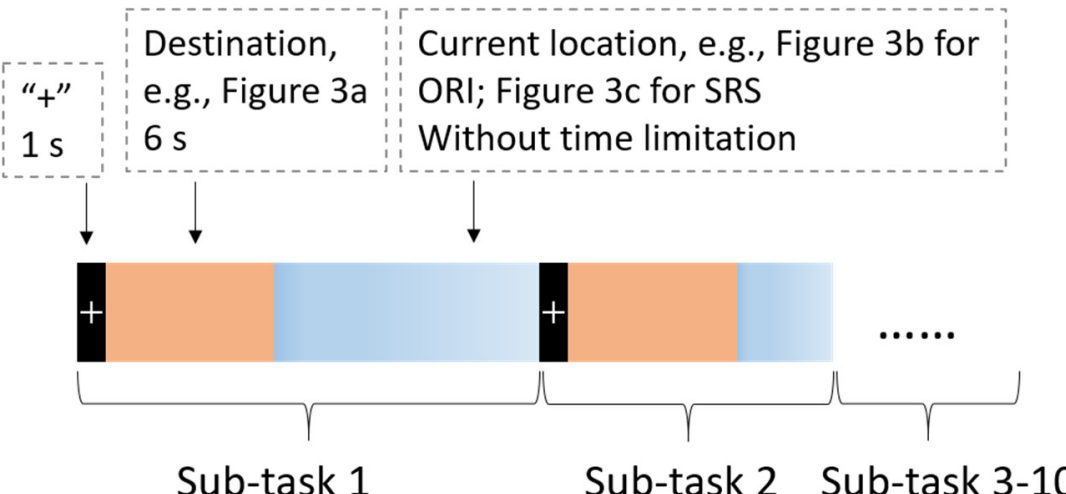

Figure 2. Sub-task design for each part.

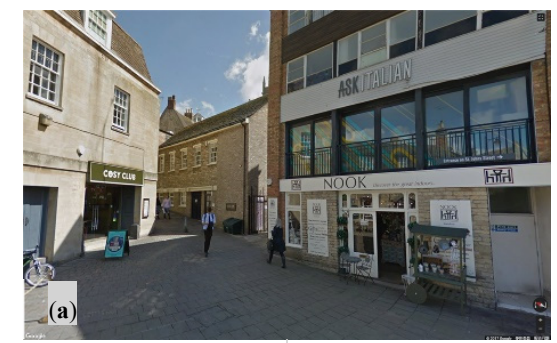

(Imagine that all scenes are observed from a first-person view, and you cannot see the origin and destination point from where you stand in the picture.) This picture was shot somewhere along the road network that you learnt yesterday and is the destination in the task.

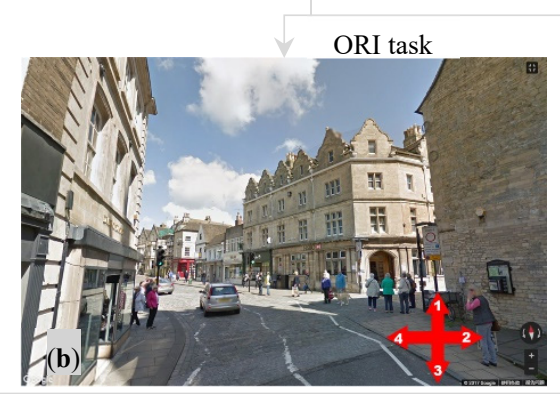

This picture was also shot along the road network that you learnt yesterday from where you stand right now (the origin). You should choose the relative direction of the destination from the four labelled arrows (1-4). If the relative direction is not exactly front/behind/left/right, please choose the closest direction.

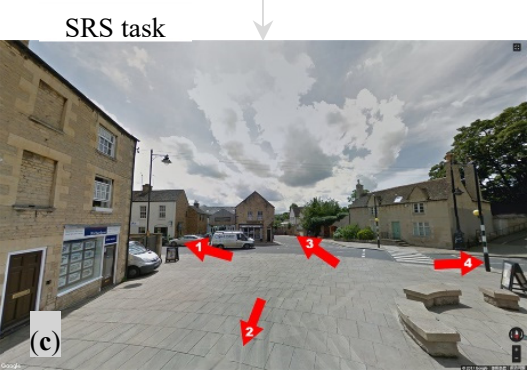

This picture was also shot in the road network that you learnt yesterday from where you stand right now (the origin). You should choose the road that leads to the shortest route to the destination from the labelled four arrows (1-4).

Figure 3. Example of experimental stimulus: (a): destination; (b): current position for orientation (ORI) task; (c): current position for shortest route selection (SRS) task. Tasks are described along the figures in grey text boxes.

\subsection{Data Analysis}

An I-VT (velocity-threshold identification) fixation filter with the default parameters in Tobii Studio was used for fixation filtering. We labelled the Road and Label areas of interest (AOIs) to conduct further analyses (Figure 4). The Road AOIs represented roads and walking areas on squares. The Label AOIs represented the signs of shops, front doors of buildings and recognizable advertisements. The areas covered by arrows were excluded because participants had to watch the arrows quite often. We used Quick Selection in Adobe Photoshop CS6 (Adobe Photoshop: https://www.photoshop.com/) to obtain the pixel number of each AOI and applied this value to represent the size of the AOI. If there were multiple Label or Road AOIs, the same kind of AOIs were aggregated into one AOI group. 


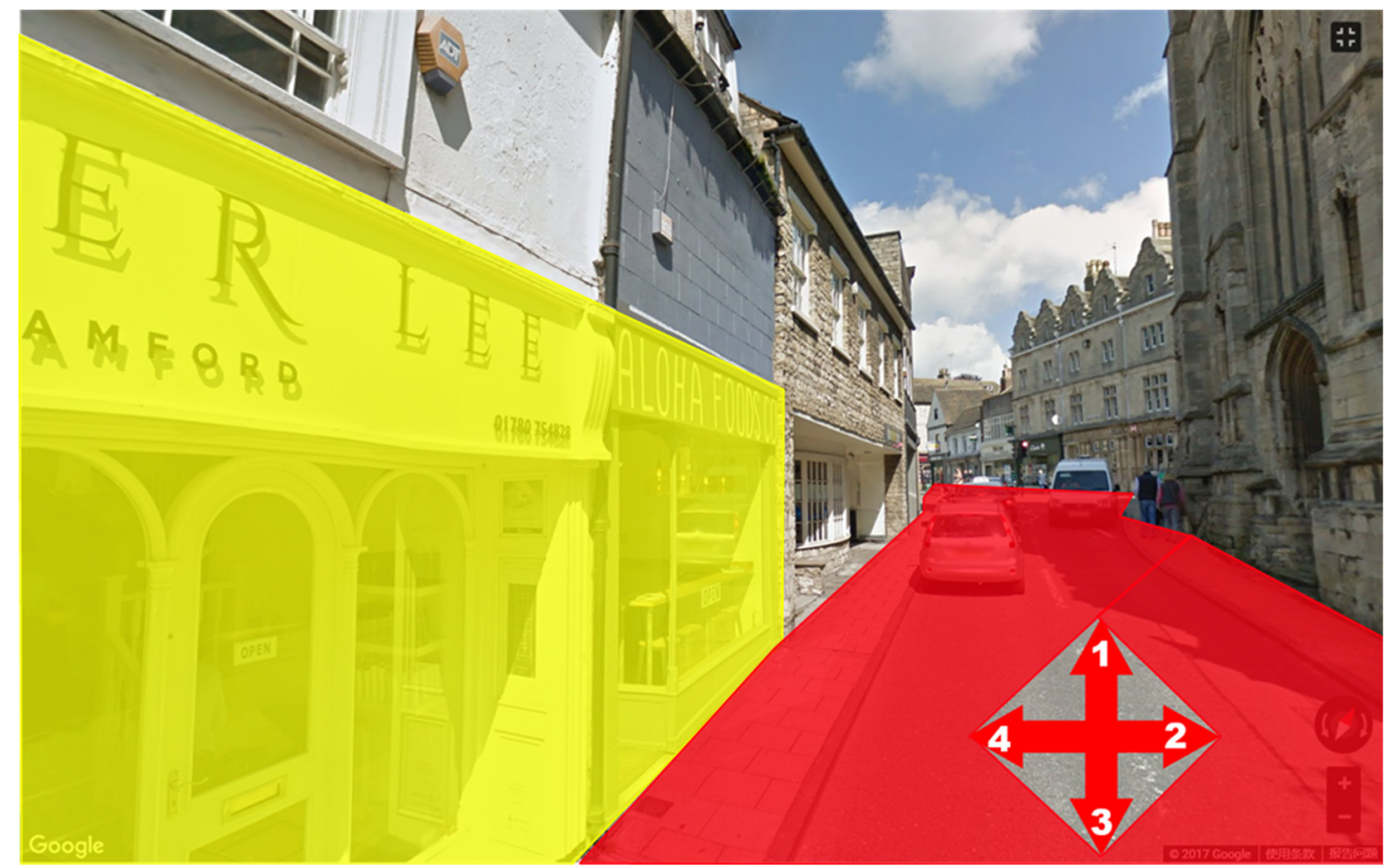

Figure 4. Areas of interest (AOI) example: the yellow part is the Label AOI and the red part is the Road AOI.

Accuracy of Answers and response time were used to represent the participants' total performance. As information is processed during fixation, fixation-based metrics, especially duration, are usually used for task-related analysis $[47,48]$. For a more detailed review of eye movement metrics and their cognitive meaning, please refer to [32,49]. Four eye movement metrics were used (Table 1): time to first fixation, where a short time to first fixation means the object quickly attracts visual attention and has strong visual guidance [50,51]; average fixation duration, where a long average fixation duration indicates high processing difficulty $[47,50,52,53]$ in pedestrian navigation; fixation count, where a high fixation count means a large processing load [54]; and fixation duration (also referred to as fixation time [47]), where a long fixation duration indicates that a long time is needed to process the information. As fixation count and fixation duration are strongly related to AOI size, we used the original value per 10,000 pixels in this analysis.

Table 1. Eye movement metrics.

\begin{tabular}{ccc}
\hline Metric & Description & Unit \\
\hline Accuracy of Answers & $\begin{array}{c}\text { Number of subtasks that were skipped, } \\
\text { misjudged or correctly completed by all } \\
\text { participants. }\end{array}$ & count \\
\hline Response Time & $\begin{array}{c}\text { Time required by participants to make a } \\
\text { decision (start with the origin point } \\
\text { shown). }\end{array}$ & $\mathrm{s}$ \\
\hline Time to First Fixation & Time spent before the AOI was first \\
fixated on. & $\mathrm{s}$ \\
\hline Fixation Duration & Total fixation duration within the AOI. & s/pixel number $\times 10,000$ \\
\hline Fixation Count & Total fixation count within the AOI. & number/pixel number $\times 10,000$ \\
\hline Average Fixation Duration & Fixation duration/Fixation count. & $\mathrm{s}$ \\
\hline
\end{tabular}

First, the outliers in the raw data were excluded based on the three-sigma rule. Second, we performed linear mixed model regression for the statistical test, as the data were based on a 
within-participant experiment and were not independent [55]. We predicted the eye movement metrics with road pattern and AOI category as fixed effects, and participants as random effect using Python's statsmodels module. We identified $p<0.01$ as a significant influence and $p>0.01$ as no significant influence.

Note that the statistical test was applied on data records based on AOIs instead of data records based on participants. Although seven samples for ORI tasks and three samples for SRS tasks were excluded, as mentioned in the Participants section, the test was performed on hundreds of data records.

\section{Results}

\subsection{Overall Performance}

Table 2 shows the accuracy of answers in different road patterns. In both the ORI task and the SRS task, participants performed better with irregular road patterns, as they skipped or misjudged fewer subtasks and made more correct choices.

Table 2. Task accuracy of answers (count).

\begin{tabular}{ccccc}
\hline Task & Road Pattern & Correct & Skipped & Incorrect \\
\hline \multirow{2}{*}{ Orientation (ORI) } & Irregular & 56 & 28 & 56 \\
& Regular & 30 & 27 & 83 \\
\hline Shortest Route & Irregular & 104 & 15 & 61 \\
Selection (SRS) & Regular & 92 & 19 & 69 \\
\hline
\end{tabular}

The response time for all the four categories of tasks ranged from approximately 8 to $15 \mathrm{~s}$. For the same kind of task, the response time does not show a considerable difference (Figure 5). The participants spent the same amount of time on the same tasks for different road patterns.

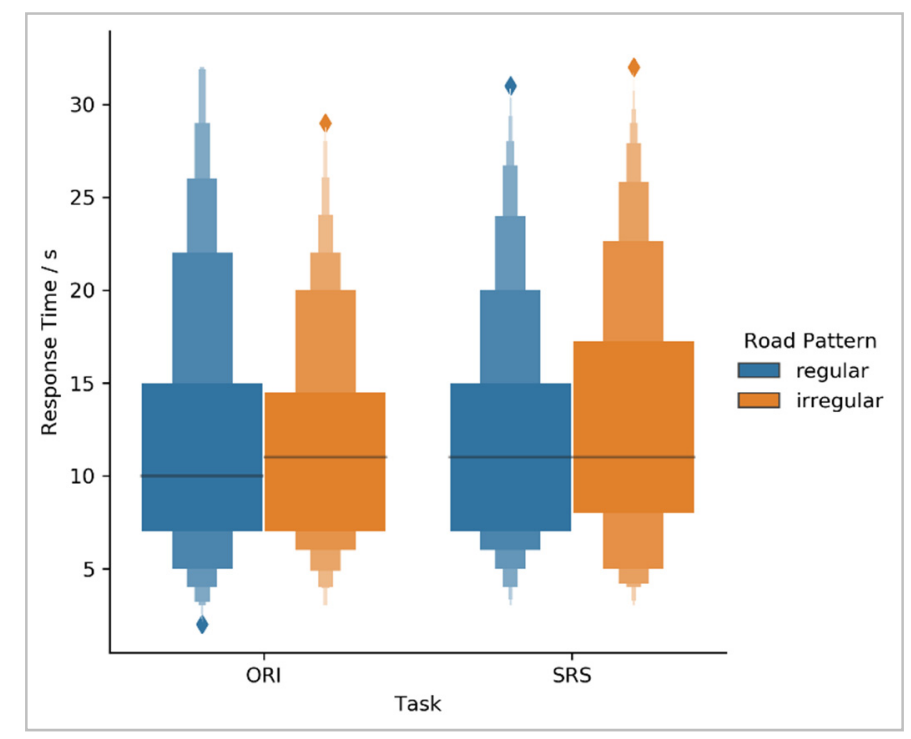

Figure 5. Statistics of response time.

\subsection{ORI Task}

\subsubsection{Time to First Fixation}

Figure 6 shows statistics of the time to first fixation in the ORI task. For both the irregular road pattern and the regular road pattern, the time to first fixation was shorter on Label AOIs than on Road AOIs (Figure 6a). Time to first fixation difference between irregular and regular road patterns in the 
ORI task are shown in Figure 6b. For the Road AOI, time to first fixation was shorter with the irregular road pattern than with the regular pattern, whereas for the Label AOI, this time was longer.

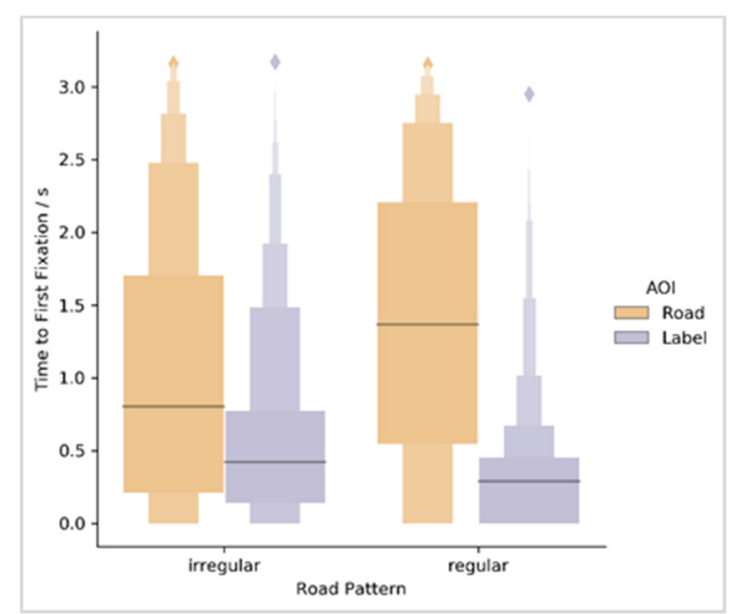

(a)

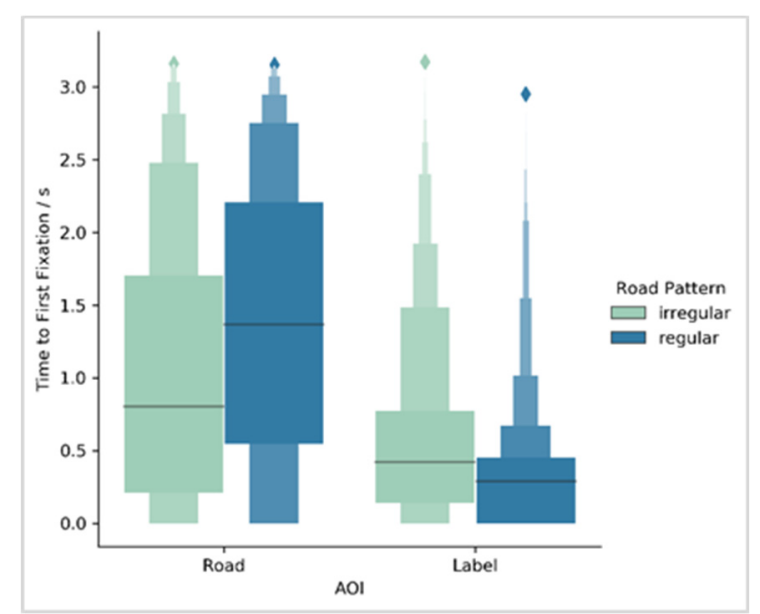

(b)

Figure 6. Statistics of the time to first fixation in the ORI task: (a): grouped by road pattern and (b): grouped by AOI category.

The results of linear mixed model regression of the time to first fixation in the ORI task are shown in Table 3. The coefficients of the road pattern and AOI category are -0.072 and -0.727 , respectively. However, only the AOI category contributes significantly to the time to first fixation in the ORI task $(p<0.01)$. This result shows that participants first fixated on the labels and then fixated on the roads in both conditions. While participants tended to fixate on the roads faster or fixate on labels more slowly for irregular road patterns than regular road patterns, the difference is not significant.

Table 3. Linear mixed model regression results of time to first fixation in the ORI task. Coeff, coefficient; $\mathrm{SE}$, standard error; MSE, mean square error; Group Var, Group Variable.

\begin{tabular}{cccccc}
\hline & Coeff & SE & $\mathbf{z}$ & $p$-Value & MSE \\
\hline Intercept & 2.034 & 0.122 & 16.635 & 0.000 & 0.5536 \\
Road Pattern & -0.072 & 0.055 & -1.315 & 0.189 & \\
AOI Category & -0.727 & 0.058 & -12.522 & 0.000 & \\
Group Var & 0.001 & 0.006 & & & \\
\hline
\end{tabular}

\subsubsection{Average Fixation Duration}

For both road patterns, the average fixation duration for the Label AOIs was longer than that for the Road AOIs, as shown in Figure 7a. For irregular road patterns, the average fixation duration for both roads and labels was longer than that for regular patterns (Figure $7 \mathrm{~b}$ ).

Table 4 shows the results of linear mixed model regression of the average fixation duration in the ORI task. The coefficients of the road pattern and AOI category are -0.013 and 0.024 , respectively. Both the road pattern and AOI category contribute significantly to the average fixation duration in the ORI task $(p<0.01)$. The processing difficulty for the Label AOIs was higher than that for the Road AOIs. Participants made more efforts to process both road and label information with irregular patterns. 


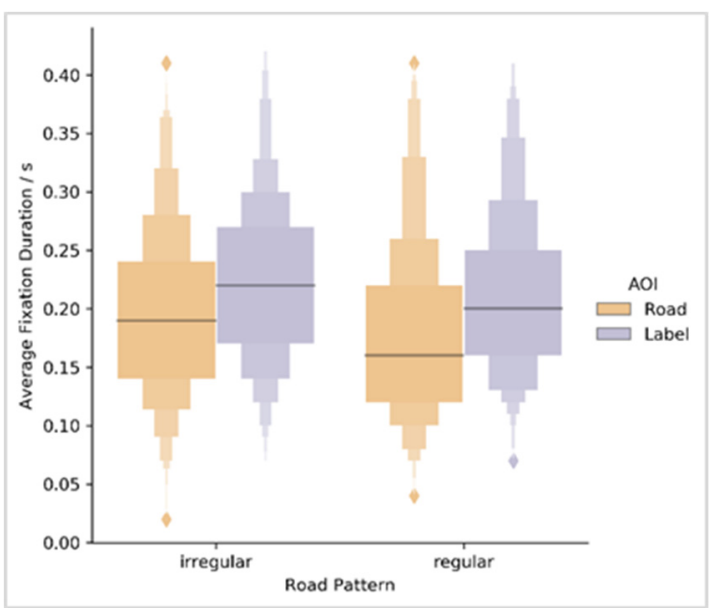

(a)

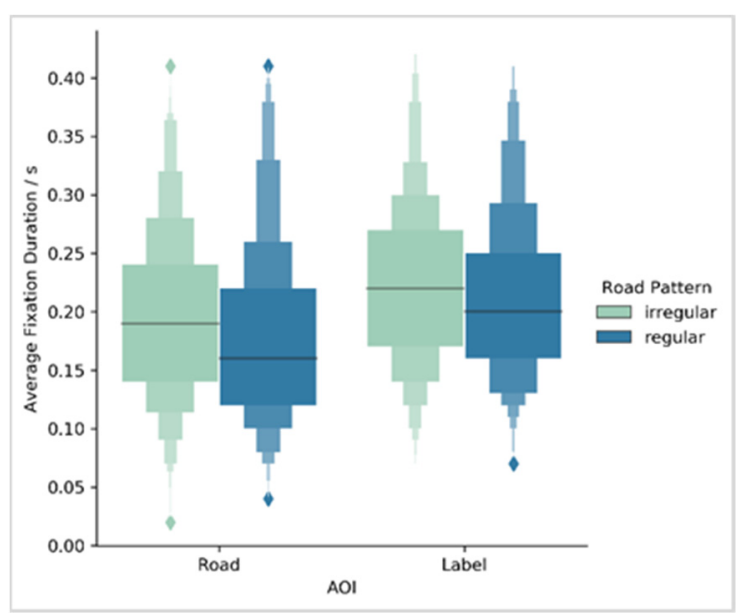

(b)

Figure 7. Statistics of average fixation duration in the ORI task: (a): grouped by road pattern and (b): grouped by AOI category.

Table 4. Linear mixed model regression results of average fixation duration in the ORI task.

\begin{tabular}{cccccc}
\hline & Coeff & SE & $\mathbf{z}$ & $p$-Value & MSE \\
\hline Intercept & 0.187 & 0.015 & 12.471 & 0.000 & 0.0037 \\
Road Pattern & -0.013 & 0.004 & -3.054 & 0.002 & \\
AOI Category & 0.024 & 0.004 & 5.795 & 0.000 & \\
Group Var & 0.002 & 0.014 & & & \\
\hline
\end{tabular}

\subsubsection{Fixation Count}

As shown in Figure 8a, the fixation count for the Label AOIs was greater than that for the Road AOIs in both patterns. The fixation count for the Road AOIs was greater for irregular road patterns than that for regular patterns, and that for the Label AOIs was smaller than that for the irregular road patterns (Figure 8b).

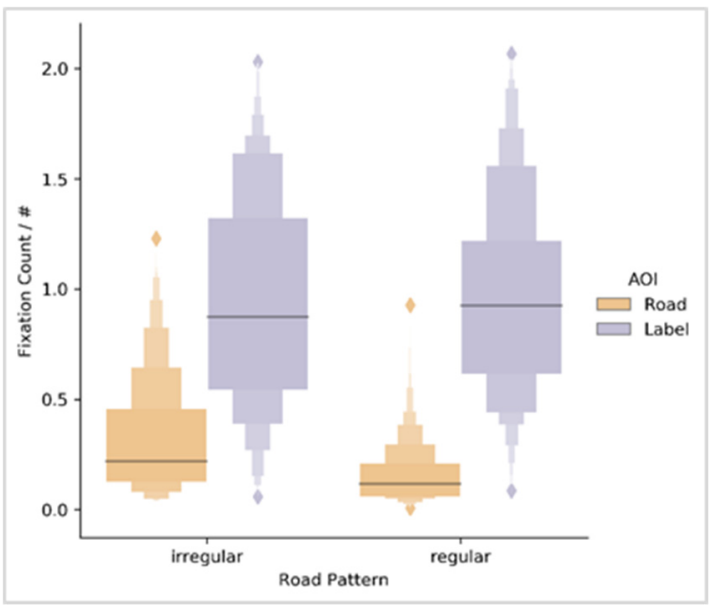

(a)

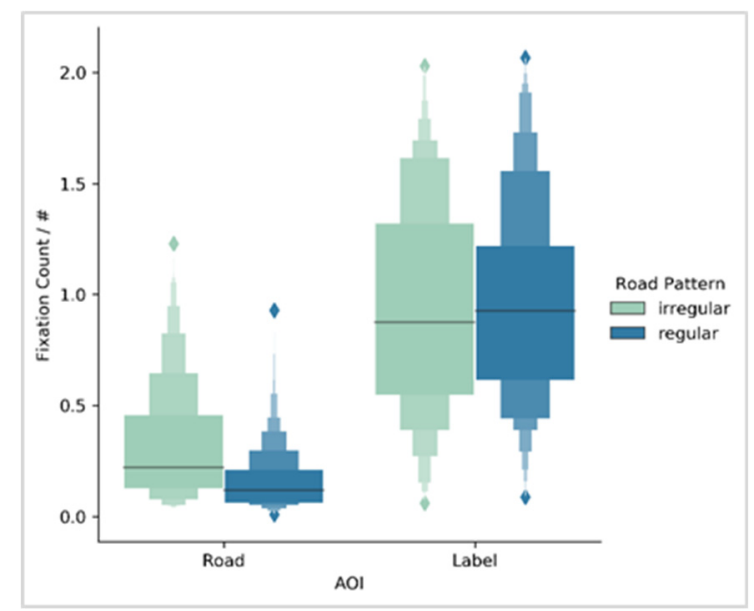

(b)

Figure 8. Statistics of fixation count in the ORI task: (a): grouped by road pattern and (b): grouped by AOI category.

Table 5 shows the linear mixed model regression results for the fixation count in the ORI task. The coefficients of the road pattern and the AOI category are -0.069 and 0.713 , respectively. Both the road pattern and the AOI category contribute significantly to the fixation count in the ORI task $(p<$ 
0.01). Participants paid more attention to the Label AOIs with irregular road patterns than those with regular road patterns. Participants paid more attention to the Road AOIs with irregular road patterns and less to the Label AOIs with irregular road patterns.

Table 5. Linear mixed model regression results of fixation count in the ORI task.

\begin{tabular}{cccccc}
\hline & Coeff & SE & $\mathbf{z}$ & $p$-Value & MSE \\
\hline Intercept & -0.371 & 0.057 & -6.497 & 0.000 & 0.1256 \\
Road Pattern & -0.069 & 0.026 & -2.653 & 0.008 & \\
AOI Category & 0.713 & 0.026 & 27.556 & 0.000 & \\
Group Var & 0.007 & 0.011 & & & \\
\hline
\end{tabular}

\subsubsection{Fixation Duration}

The fixation duration shows that the fixation duration for the Label AOIs was greater than that for the Road AOIs for both patterns (Figure 9a). The fixation count for the Road AOIs with irregular road patterns was greater than those with regular patterns, but the fixation durations for the Label AOIs with irregular and regular patterns were similar (Figure 9b).

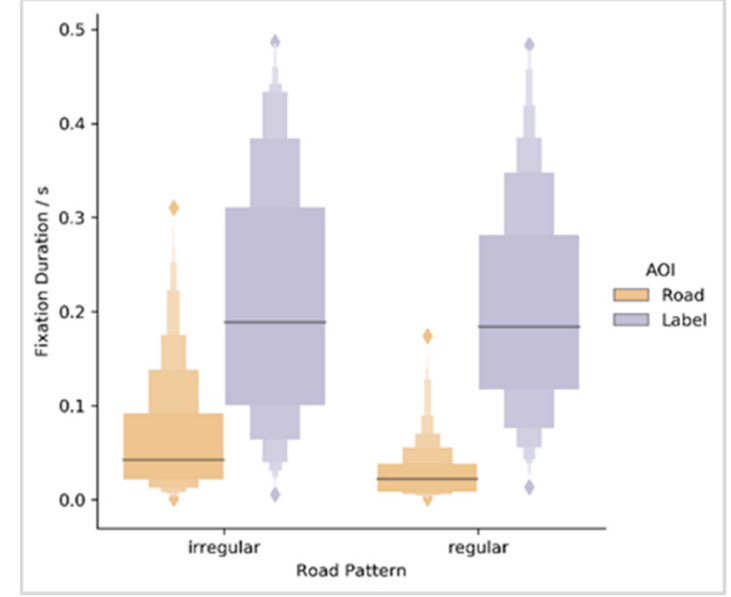

(a)

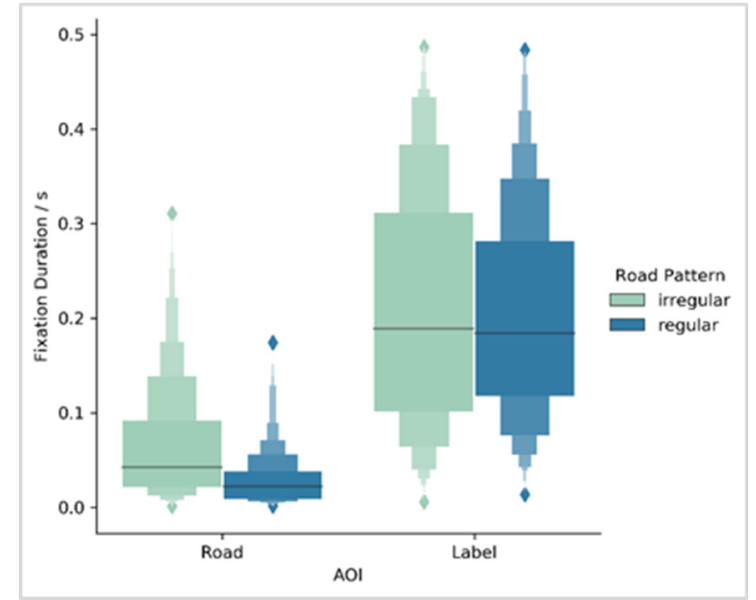

(b)

Figure 9. Statistics of fixation duration in the ORI task: (a): grouped by road pattern and (b): grouped by AOI category.

As shown in Table 6, the coefficients of the road pattern and the AOI category are -0.022 and 0.160 , respectively. Both factors contribute significantly to the fixation duration $(p<0.01)$. Participants paid more attention to the Label AOIs. As the fixation durations for the Label AOIs are almost the same for irregular and regular patterns, we assume the influence of the AOI category results from the Road AOIs.

Table 6. Linear mixed model regression results of fixation duration in the ORI task.

\begin{tabular}{cccccc}
\hline & Coeff & SE & $\mathbf{z}$ & $p$-Value & MSE \\
\hline Intercept & -0.078 & 0.016 & -4.987 & 0.000 & 0.0073 \\
Road Pattern & -0.022 & 0.006 & -3.509 & 0.000 & \\
AOI Category & 0.160 & 0.006 & 25.693 & 0.000 & \\
Group Var & 0.001 & 0.006 & & & \\
\hline
\end{tabular}




\subsection{SRS Task}

\subsubsection{Time to First Fixation}

The statistics of the time to first fixation in the SRS task are shown in Figure 10. As shown in Figure 10a, with irregular and regular road patterns, the time to first fixation for the Label AOIs was shorter than that for the Road AOIs. As shown in Figure 10b for the Road AOIs, the time to first fixation was shorter for irregular road patterns than for regular patterns. For the Label AOIs, the time to first fixation was slightly longer for irregular road patterns.

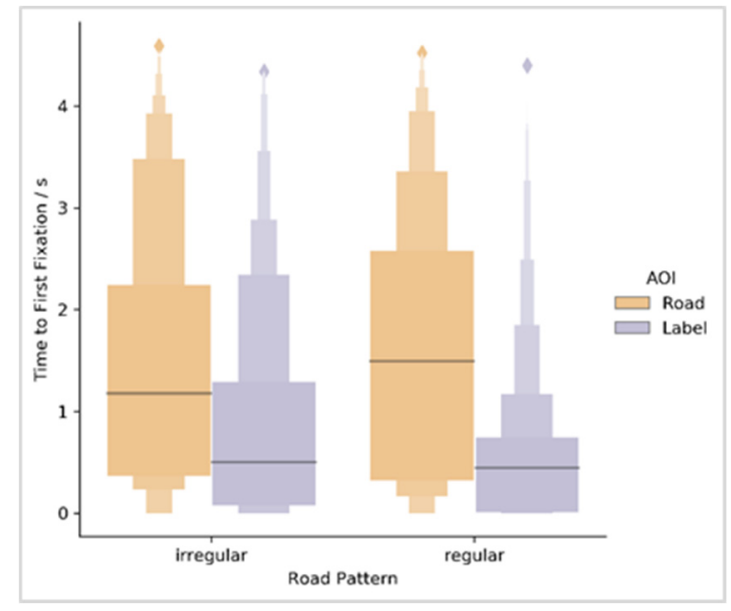

(a)

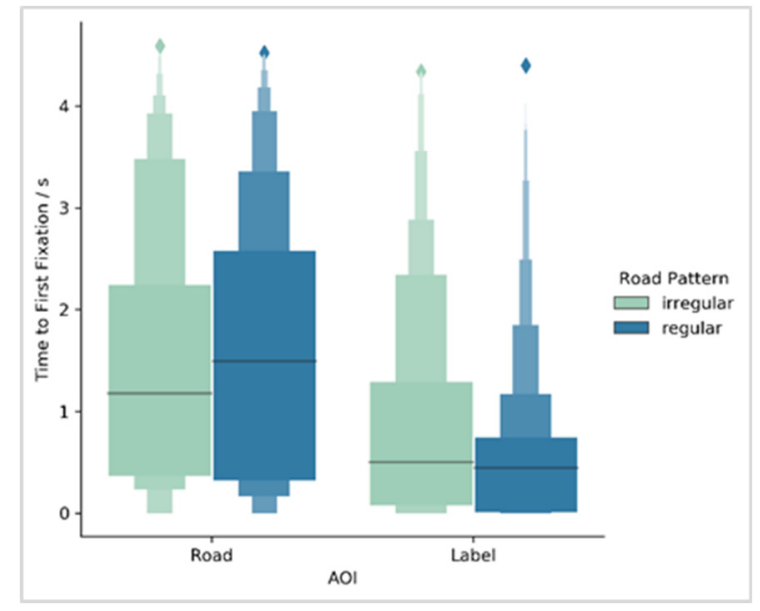

(b)

Figure 10. Statistics of time to first fixation in the SRS task: (a): grouped by road pattern and (b): grouped by AOI category.

Table 7 shows the results of the linear mixed model regression for the time to first fixation in the SRS task. The coefficients of the road pattern and the AOI category are -0.100 and -0.812 , respectively. However, the road pattern's contribution is not significant $(p=0.131)$. The time to first fixation in the SRS task is influenced only by the AOI category. The participants performed similarly as in the ORI tasks, in that they first fixated on the labels and then fixated on the roads.

Table 7. Linear mixed model regression results of time to first fixation in the SRS task.

\begin{tabular}{cccccc}
\hline & Coeff & SE & $\mathbf{z}$ & $p$-Value & MSE \\
\hline Intercept & 2.502 & 0.142 & 17.644 & 0.000 & 1.1803 \\
Road Pattern & -0.100 & 0.066 & -1.511 & 0.131 & \\
AOI Category & -0.812 & 0.066 & -12.23 & 0.000 & \\
Group Var & 0.016 & 0.011 & & & \\
\hline
\end{tabular}

\subsubsection{Average Fixation Duration}

As shown in Figure 11a, the average fixation duration for the Label AOIs was longer than that for the Road AOIs for both irregular road patterns and regular patterns. As shown in Figure 11b, for the Road AOIs, the average fixation duration for the irregular road patterns was longer than that for the regular road patterns. For the Label AOIs, the average fixation durations for the roads and labels were similar. 


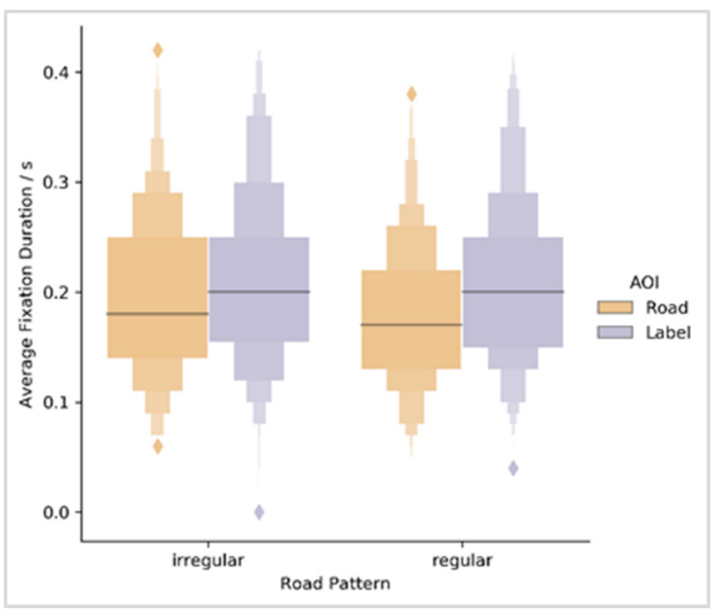

(a)

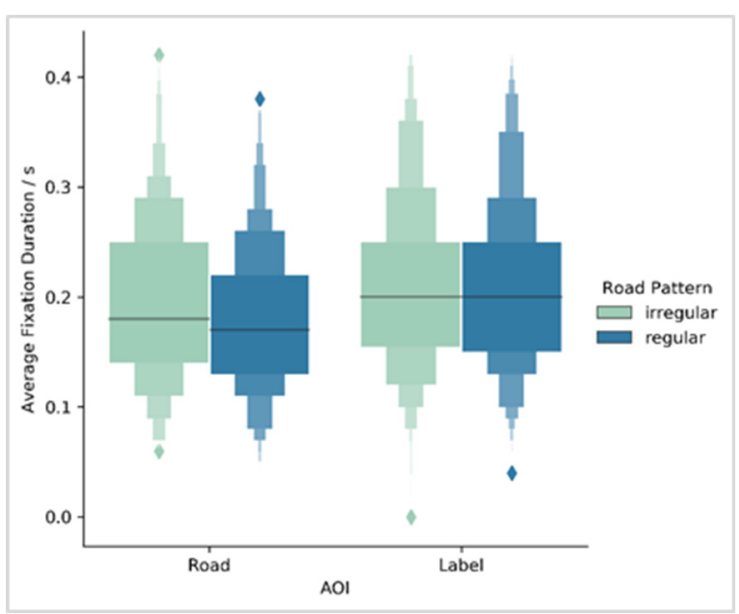

(b)

Figure 11. Statistics of average fixation duration in the SRS task: (a): grouped by road pattern and (b): grouped by AOI category.

Table 8 shows the results of the linear mixed model regression of the average fixation duration in the SRS task. The coefficients of the road pattern and AOI category are -0.009 and 0.021 , respectively. Both factors contribute significantly $(p<0.01)$. The average fixation duration in the SRS task is influenced by both the road pattern and the AOI category. As in the ORI tasks, the processing difficulty was higher for the Label AOIs. Similar to different road patterns, the average fixation duration is the same for the Label AOIs. We assume that the influence of road pattern is derived from the Road AOIs. Processing Road AOI information with irregular road patterns is more difficult than that with regular road patterns.

Table 8. Linear mixed model regression results of average fixation duration in the SRS task.

\begin{tabular}{cccccc}
\hline & Coeff & SE & $\mathbf{z}$ & $p$-Value & MSE \\
\hline Intercept & 0.182 & 0.014 & 13.261 & 0.000 & 0.0034 \\
Road Pattern & -0.009 & 0.003 & -2.604 & 0.009 & \\
AOI Category & 0.021 & 0.003 & 6.170 & 0.000 & \\
Group Var & 0.002 & 0.015 & & & \\
\hline
\end{tabular}

\subsubsection{Fixation Count}

Figure 12a shows that the fixation count for the Label AOIs was greater than that for the Road AOIs with both patterns. Figure $12 \mathrm{~b}$ shows that the fixation count for both Road AOIs and Label AOIs with irregular road patterns was greater than that with regular road patterns.

Table 9 shows that the coefficients of the road pattern and the AOI category are -0.006 and 1.346, respectively. Only the AOI category contributes significantly $(p<0.01)$. The fixation count in the SRS task is influenced only by the AOI category. Similar to the ORI task, participants paid more attention to the Label AOIs with both road patterns. 


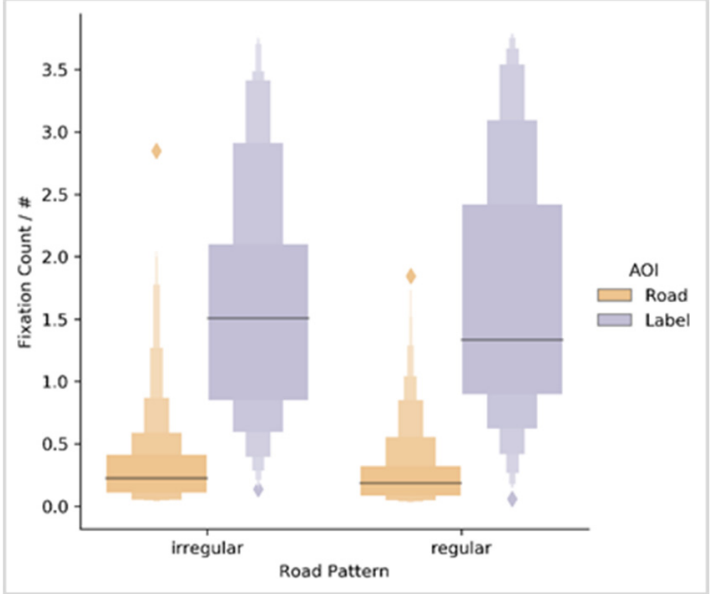

(a)

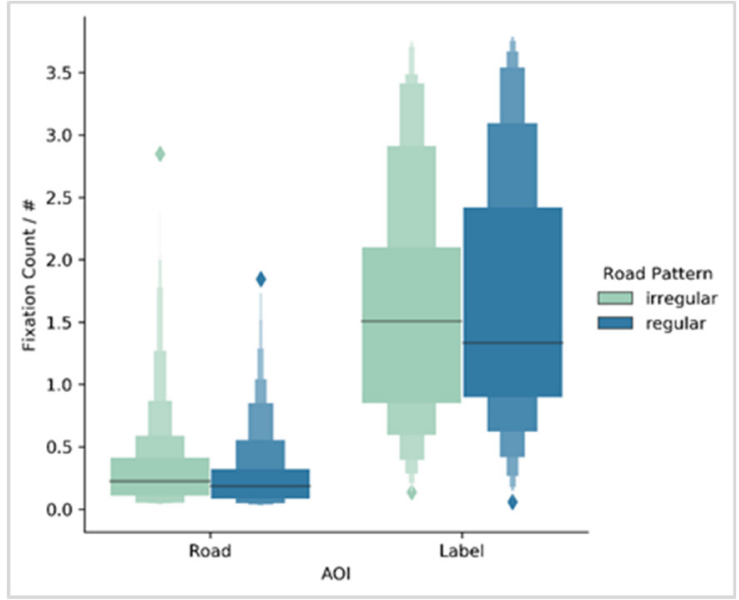

(b)

Figure 12. Statistics of fixation count in the ORI task: (a): grouped by road pattern and (b): grouped by AOI category.

Table 9. Linear mixed model regression results of fixation count in the SRS task.

\begin{tabular}{cccccc}
\hline & Coeff & SE & $\mathbf{z}$ & $p$-Value & MSE \\
\hline Intercept & -1.030 & 0.091 & -11.293 & 0.000 & 0.4805 \\
Road Pattern & -0.006 & 0.043 & -0.150 & 0.881 & \\
AOI Category & 1.346 & 0.043 & 31.363 & 0.000 & \\
Group Var & 0.009 & 0.009 & & & \\
\hline
\end{tabular}

\subsubsection{Fixation Duration}

Figure 13a shows that the fixation duration for the Label AOIs was longer than that for the Road AOIs with both patterns. Figure 13b shows that the fixation duration for both Road AOIs and Label AOIs was longer for irregular road patterns than for regular patterns.

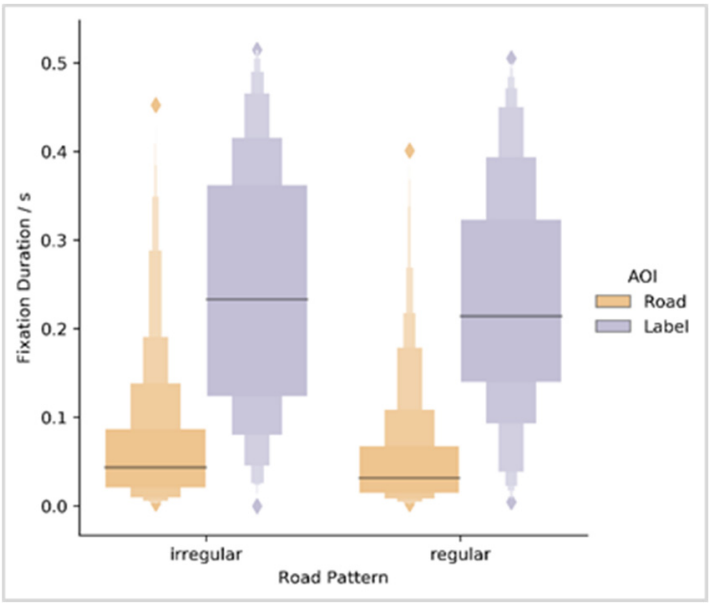

(a)

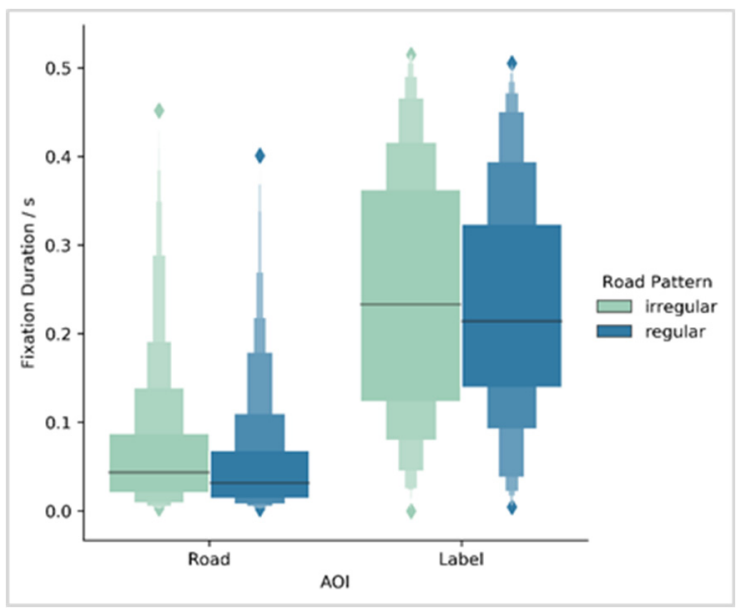

(b)

Figure 13. Statistics of fixation duration in the SRS task: (a): grouped by road pattern and (b): grouped by AOI category.

Table 10 shows that the coefficients of the road pattern and AOI category are -0.013 and 0.180 , respectively. AOI category contributes significantly $(p<0.01)$ and road pattern's contribution is less significant $(p=0.031)$. The fixation count in the SRS task is influenced by the AOI category. Participants paid more attention to the Label AOIs with both road patterns. Although the participants tended to 
pay more attention for both the Road AOIs and the Label AOIs to perform tasks with irregular road patterns than tasks with regular road patterns, as in ORI tasks, the difference is not significant.

Table 10. Linear mixed model regression results of fixation duration in the SRS task.

\begin{tabular}{cccccc}
\hline & Coeff & SE & $\mathbf{z}$ & $p$-Value & MSE \\
\hline Intercept & -0.099 & 0.014 & -6.909 & 0.000 & 0.0087 \\
Road Pattern & -0.013 & 0.006 & -2.156 & 0.031 & \\
AOI Category & 0.180 & 0.006 & 28.627 & 0.000 & \\
Group Var & 0.001 & 0.004 & & & \\
\hline
\end{tabular}

\section{Discussion}

\subsection{Performance on Road and Label AOIs}

This study shows that for both ORI and SRS tasks, participants first fixated on Label AOIs and then on Road AOIs with both irregular and regular road patterns, and they had more fixation counts and longer fixation durations per 10,000 pixels for Label AOIs than for Road AOIs. They also had longer average fixation durations for Label AOIs than for Road AOIs. Labels tended to grab participants' attention faster or receive more attention, and they also required more time to process.

This universal difference between Label and Road AOIs in this study is not surprising. The Road AOIs in this study only show road trends and intersections, and in regular road patterns, they are highly similar, whereas Label AOIs vary in shape, colour and texture and have semantic meaning. Therefore, the Label AOIs have a higher degree of recognition [56] and are more likely to be regarded as landmarks. This finding is consistent with that of Liao and Dong [57], who found that displaying 3D models, which are more salient than 2D maps, can improve map usability for male users. In general, rich label information should be provided for ORI and SRS tasks, and navigation systems should highlight the label information, for example, by using larger annotation or bright colours. On the other hand, with ORI tasks in irregular road patterns, participants also tried to rely on roads because the difference of average fixation duration was only marginally significant. This finding indicates that unique road patterns can also help wayfinding. Pedestrians may pay more attention to roads if the roads vary in texture or have some semantic meaning [56], for example, if traffic signs are painted on roads, especially on roads with unique patterns.

\subsection{Performance in Different Road Patterns}

Participants performed better with the irregular road pattern than the regular pattern for both tasks because they made more correct choices within the same amount of time. Since we chose areas with similar building styles, it could be concluded that the difference in the road pattern is the main cause of the performance difference. While Hirtle et al. [58] stated that oblique intersections can cause disorientation, this study shows that irregular road patterns with curvatures are better remembered. The unique intersections in irregular road patterns provide richer and more helpful information. The better performance for irregular road patterns may also explain pedestrians' preference for curvy roads in previous research [25].

The gaze data indicate that the road pattern's influence is caused by both roads and labels but is more related to roads. For both tasks, road pattern did not influence the time to first fixation for the Road AOI or Label AOI. For the ORI tasks, compared with the regular road pattern, participants had a longer average fixation for both Road AOIs and Label AOIs, a greater fixation count for the Road AOIs and a smaller fixation count for the Label AOIs with irregular patterns. The difference in the fixation duration was only observed in the Road AOIs; participants had a longer fixation duration for the Road AOIs with the irregular patterns. These differences indicate that ORI tasks with irregular road patterns are more demanding and are consistent with the findings of Liu et al. [31] based on fMRI. These authors found that when performing orientation tasks, the participants showed more activation 
in the functional brain areas that were related to decision-making (middle frontal gyrus and medial frontal gyrus) and eye movement (superior frontal gyrus) with an irregular road pattern than a regular pattern. In the SRS tasks, the difference in the average fixation duration between the road patterns was only shown for the Road AOIs, and participants had a longer average fixation duration for the Road AOIs with irregular road patterns. This difference indicates that for SRS tasks with irregular road patterns, roads were more likely to provide more information than they did for SRS tasks with regular road patterns. In both the ORI and SRS tasks, participants paid more attention to roads with irregular road patterns, where the roads had unique intersections or turns. Although colour, texture or semantic differences among the different roads were not observed, the roads provided important information based on the structure, which is consistent with the results obtained by Hirtle et al. [58], who found that unique intersections can be regarded as landmarks. Therefore, for navigation purposes, highlighting road patterns by indicating turns, intersections and curvatures could be helpful. This result encourages the construction of more unique intersections or irregular roads.

As Gibson indicated (summarized by Kitchin and Blades [59]), transitions (i.e., where the view changes considerably) are important for successful wayfinding. Transitions happen either when the pedestrian walks past a previous vista or when there is a turn. In regular road patterns, pedestrians always navigated forward and there were always sharp turns. However, in irregular road patterns, pedestrians might adjust the moving direction, which could be a hint about their location. In addition, with non-sharp turns, some of the buildings in previous views could remain visible after the transitions and help the pedestrian to orientate. The results show that people identify transitions mainly based on buildings, although the roads themselves are also important information sources regarding the transition for orientation. Thus, with irregular road patterns where the transitions are mostly unique, people tend to pay more attention to the roads than they do with regular road patterns.

\subsection{Limitations}

We identify some limitations that could be improved in future studies. The materials used in this study were street views from Google Maps, which were joint street views taken at different times. The inconsistencies of some shops may have caused confusion and further influenced the participants' performances. Although there were only a few inconsistencies in the study area, the results could be improved if the environment is properly controlled (e.g., in a virtual reality environment). People may also act differently in the 3D physical world compared with a highly controlled lab environment. For example, if there are people walking around, the participants may pay much more attention to the faces. People may also walk around the origin and gather information from different directions in the physical world. In addition, the experiment reported here was conducted in a fixed irregular-regular order, and an even better performance might be obtained for irregular roads if the order was counterbalanced because of learning effects. Testing how this difference changes as people become more familiar with the road network is an interesting future research direction.

\section{Conclusions and Future Work}

In this study, we aimed to identify whether gaze differences occurred between regular and irregular road patterns during orientation and route selection. We conducted an experiment in which 21 participants were asked to determine the relative orientation and choose the shortest route based on eye-tracking technology. We found that the performance was better for irregular road patterns than regular patterns. For both regular patterns and irregular patterns, labels provided the participants with more information, and the influence of the road pattern on the gaze was greater on roads than labels in both tasks. Participants tended to rely more on roads with irregular road patterns than those with regular patterns. The results contribute to further understanding the influence of road patterns on geospatial cognition and indicate that labels and unique road intersections or turns should be highlighted to support wayfinding and navigation tasks. 
The results may have a limited ability to explain the influence of road patterns because the experiment was conducted on a desktop computer in a laboratory environment and based on newly learned road networks. Thus, the results may be improved by further investigation based on immersive or physical environments and on more familiar road networks.

Author Contributions: Conceptualization, Bing Liu and Weihua Dong; Data Curation, Bing Liu and Zhicheng Zhan; Formal Analysis, Bing Liu; Methodology, Bing Liu, Zhicheng Zhan and Shengkai Wang; Writing-Original Draft, Bing Liu; Writing-Review and Editing, Bing Liu, Weihua Dong, Zhicheng Zhan, Shengkai Wang and Liqiu Meng; Funding Acquisition, Weihua Dong; Project Administration, Weihua Dong; Supervision, Weihua Dong. All authors have read and agreed to the published version of the manuscript.

Funding: This research is supported by the National Natural Science Foundation of China (NSFC, Grant No. 41871366) and the China Scholarship Council (Grant No. 201806040219).

Acknowledgments: The authors would like to thank the editor and anonymous reviewers for their efforts to review this paper. We also thank Qiliang Liu (Central South University, China) for the helpful discussions and Bin Yu (Beijing Normal University, China) for the technical help.

Conflicts of Interest: The authors declare no conflict of interest.

Data Availability Statement: The datasets generated and analysed during the current study are not publicly available as they contain information that could compromise privacy and consent of research participants, but they are available from the corresponding author on reasonable request.

\section{References}

1. Xie, F.; Levinson, D. Measuring the Structure of Road Networks. Geogr. Anal. 2007, 39, 336-356. [CrossRef]

2. Parthasarathi, P.; Levinson, D.; Hochmair, H. Network structure and travel time perception. PLoS ONE 2013, 8, e77718. [CrossRef] [PubMed]

3. Li, H.; Hu, M.; Huang, Y. Automatic Identification of Overpass Structures: A Method of Deep Learning. ISPRS Int. J. Geo-Inf. 2019, 8, 421. [CrossRef]

4. Wang, C.; Quddus, M.A.; Ison, S.G. The effect of traffic and road characteristics on road safety: A review and future research direction. Saf. Sci. 2013, 57, 264-275. [CrossRef]

5. Duranton, G.; Puga, D. Chapter 8-Urban Land Use. In Handbook of Regional and Urban Economics; Duranton, G., Henderson, J.V., Strange, W.C., Eds.; Elsevier: Amsterdam, The Netherlands, 2015; Volume 5, pp. 467-560.

6. Ben-Bassat, T.; Shinar, D. Effect of shoulder width, guardrail and roadway geometry on driver perception and behavior. Accid. Anal. Prev. 2011, 43, 2142-2152. [CrossRef] [PubMed]

7. Li, Z.; Wang, W.; Liu, P.; Ragland, D.R. Physical environments influencing bicyclists' perception of comfort on separated and on-street bicycle facilities. Transp. Res. Part D Transp. Environ. 2012, 17, 256-261. [CrossRef]

8. Bella, F. Driver perception of roadside configurations on two-lane rural roads: Effects on speed and lateral placement. Accid. Anal. Prev. 2013, 50, 251-262. [CrossRef]

9. Gargoum, S.A.; El-Basyouny, K.; Kim, A. Towards setting credible speed limits: Identifying factors that affect driver compliance on urban roads. Accid. Anal. Prev. 2016, 95, 138-148. [CrossRef]

10. Xu, J.; Luo, X.; Shao, Y.-M. Vehicle trajectory at curved sections of two-lane mountain roads: A field study under natural driving conditions. Eur. Transp. Res. Rev. 2018, 10, 12. [CrossRef]

11. Akasaka, Y.; Onisawa, T. Personalized pedestrian navigation system with subjective preference based route selection. In Intelligent Decision and Policy Making Support Systems; Springer: Heidelberg, Germany, 2008; pp. 73-91.

12. Chakraborty, B.; Hashimoto, T. Computational framework for subjective preference based route selection in pedestrian navigation system. In Proceedings of the TENCON 2011-2011 IEEE Region 10 Conference, Bali, Indonesia, 21-24 November 2011; pp. 1144-1148.

13. Meng, L.; Hu, Z.; Huang, C.; Zhang, W.; Jia, T. Optimized Route Selection Method based on the Turns of Road Intersections: A Case Study on Oversized Cargo Transportation. ISPRS Int. J. Geo-Inf. 2015, 4, 2428. [CrossRef]

14. Schroder, C.J.; Mackaness, W.A.; Gittings, B.M. Giving the 'Right' Route Directions: The Requirements for Pedestrian Navigation Systems. Trans. GIS 2011, 15, 419-438. [CrossRef] 
15. Brunyé, T.T.; Collier, Z.A.; Cantelon, J.; Holmes, A.; Wood, M.D.; Linkov, I.; Taylor, H.A. Strategies for selecting routes through real-world environments: Relative topography, initial route straightness, and cardinal direction. PLOS ONE 2015, 10, e0124404. [CrossRef]

16. Zhu, S.; Levinson, D. Do People Use the Shortest Path? An Empirical Test of Wardrop's First Principle. PLOS ONE 2015, 10, e0134322. [CrossRef]

17. Marshall, W.E.; Garrick, N.W. Street network types and road safety: A study of 24 California cities. Urban Des. Int. 2010, 15, 133-147. [CrossRef]

18. Jacob, A.; Jinesh, K.; Akkara, J.; Therattil, J.P. Effect of rural highway geometry on driver workload: A step towards safety. In Proceedings of the Emerging Trends in Engineering, Science and Technology for Society, Energy and Environment: Proceedings of the International Conference in Emerging Trends in Engineering, Science and Technology (ICETEST 2018), Thrissur, Kerala, India, 18-20 January 2018; p. 149. [CrossRef]

19. Green, P. Driver Workload as a Function of Road Geometry: A Pilot Experiment. Available online: https://deepblue.lib.umich.edu/bitstream/handle/2027.42/1046/86296.0001.001.pdf?sequence=2 (accessed on 28 March 2018).

20. Haynes, R.; Jones, A.; Kennedy, V.; Harvey, I.; Jewell, T. District Variations in Road Curvature in England and Wales and their Association with Road-Traffic Crashes. Environ. Plan. A 2007, 39, 1222-1237. [CrossRef]

21. Zhang, Y.; Bigham, J.; Ragland, D.; Chen, X. Investigating the associations between road network structure and non-motorist accidents. J. Transp. Geogr. 2014, 42, 34-47. [CrossRef]

22. Papinski, D.; Scott, D.M.; Doherty, S.T. Exploring the route choice decision-making process: A comparison of planned and observed routes obtained using person-based GPS. Transp. Res. Part F Traffic Psychol. Behav. 2009, 12, 347-358. [CrossRef]

23. Kim, S.; Choi, J.; Kim, Y. Determining the sidewalk pavement width by using pedestrian discomfort levels and movement characteristics. KSCE J. Civ. Eng. 2011, 15, 883-889. [CrossRef]

24. Ozbil, A.; Argin, G.; Yesiltepe, D. Pedestrian route choice by elementary school students: The role of street network configuration and pedestrian quality attributes in walking to school. Int. J. Des. Creat. Innov. 2016, 4, 1-18. [CrossRef]

25. D'Acci, L. Aesthetical cognitive perceptions of urban street form. Pedestrian preferences towards straight or curvy route shapes. J. Urban Des. 2019, 24, 1-17. [CrossRef]

26. Bailenson, J.N.; Shum, M.S.; Uttal, D.H. Road Climbing: Principles Governing Asymmetric Route Choices on Maps. J. Environ. Psychol. 1998, 18, 251-264. [CrossRef]

27. Hölscher, C.; Tenbrink, T.; Wiener, J.M. Would you follow your own route description? Cognitive strategies in urban route planning. Cognition 2011, 121, 228-247. [CrossRef]

28. Hochmair, H.H.; Karlsson, V. Investigation of preference between the least-angle strategy and the initial segment strategy for route selection in unknown environments. In Proceedings of the International Conference on Spatial Cognition, Bavaria, Germany, 11-13 October 2004; pp. 79-97.

29. Byrne, R.W. Memory for Urban Geography. Q. J. Exp. Psychol. 1979, 31, 147-154. [CrossRef]

30. Montello, D.R. Spatial Orientation and the Angularity of Urban RoutesA Field Study. Environ. Behav. 1991, 23, 47-69. [CrossRef]

31. Liu, B.; Dong, W.; Zhu, L.; Liu, H.; Meng, L. Using fMRI to Explore the Influence of Road Network Patterns on Geospatial Cognition. Proc. Int. Cartogr. Assoc. 2019, 2, 75. [CrossRef]

32. Giannopoulos, I.; Raubal, M.; Duchowski, A. Eye tracking for spatial research: Cognition, computation, challenges. Spat. Cogn. Comput. 2017, 17, 1-19. [CrossRef]

33. Liu, B.; Dong, W.; Meng, L. Using Eye Tracking to Explore the Guidance and Constancy of Visual Variables in 3D Visualization. ISPRS Int. J. Geo-Inf. 2017, 6, 274. [CrossRef]

34. Liao, H.; Dong, W.; Peng, C.; Liu, H. Exploring differences of visual attention in pedestrian navigation when using 2D maps and 3D geo-browsers. Cartogr. Geogr. Inf. Sci. 2017, 44, 474-490. [CrossRef]

35. Ooms, K.; De Maeyer, P.; Fack, V.; Van Assche, E.; Witlox, F. Interpreting maps through the eyes of expert and novice users. Int. J. Geogr. Inf. Sci. 2012, 26, 1773-1788. [CrossRef]

36. Andersen, N.E.; Dahmani, L.; Konishi, K.; Bohbot, V.D. Eye tracking, strategies, and sex differences in virtual navigation. Neurobiol. Learn. Mem. 2012, 97, 81-89. [CrossRef]

37. Bauer, C.; Ullmann, M.; Ludwig, B. Evaluating Indoor Pedestrian Navigation Interfaces Using Mobile Eye Tracking. 2016. Available online: https://www.researchgate.net/publication/306123898_Evaluating_Indoor_ Pedestrian_Navigation_Interfaces_Using_Mobile_Eye_Tracking (accessed on 22 May 2019). 
38. Fotios, S.; Uttley, J.; Cheal, C.; Hara, N. Using eye-tracking to identify pedestrians' critical visual tasks, Part 1. Dual task approach. Lighting Res. Technol. 2015, 47, 133-148. [CrossRef]

39. Hepperle, L.; von Stülpnagel, R. Gaze behavior during incidental and intentional navigation in an outdoor environment AU-Wenczel, Flora. Spat. Cogn. Comput. 2017, 17, 121-142. [CrossRef]

40. Liao, H.; Dong, W.; Huang, H.; Gartner, G.; Liu, H. Inferring user tasks in pedestrian navigation from eye movement data in real-world environments. Int. J. Geogr. Inf. Sci. 2019, 33, 739-763. [CrossRef]

41. Kitazawa, K.; Fujiyama, T. Pedestrian Vision and Collision Avoidance Behavior: Investigation of the Information Process Space of Pedestrians Using an Eye Tracker; Springer: Heidelberg, Germany, 2009; pp. 95-108.

42. Trefzger, M.; Blascheck, T.; Raschke, M.; Hausmann, S.; Schlegel, T. A Visual Comparison of Gaze Behavior from Pedestrians and Cyclists. In Proceedings of the ETRA 2018-Symposium on Eye Tracking Research and Applications, Warsaw, Poland, 14 June 2018; pp. 1-5.

43. Giannopoulos, I.; Kiefer, P.; Raubal, M. GazeNav: Gaze-Based Pedestrian Navigation. In Proceedings of the 17th International Conference on Human-Computer Interaction with Mobile Devices and Services, Copenhagen, Denmark, 24-27 August 2015; pp. 337-346.

44. Kitchin, R.; Blades, M. The Cognition of Geographic Space; I.B.Tauris: London, UK, 2002; p. 7.

45. Golledge, R.G. The Nature of Geographic Knowledge. Ann. Assoc. Am. Geogr. 2002, 92, 1-14. [CrossRef]

46. Google. Google Maps JavaScript API. Available online: https://developers.google.com/maps/documentation/ javascript/tutorial (accessed on 1 October 2017).

47. De Cock, L.; Viaene, P.; Ooms, K.; Van de Weghe, N.; Michels, R.; Wulf, A.; Vanhaeren, N.; De Maeyer, P. Comparing written and photo-based indoor wayfinding instructions through eye fixation measures and user ratings as mental effort assessments. J. Eye Mov. Res. 2019, 12, 1.

48. Coltekin, A.; Heil, B.; Garlandini, S.; Fabrikant, S.I. Evaluating the effectiveness of interactive map interface designs: A case study integrating usability metrics with eye-movement analysis. Cartogr. Geogr. Inf. Sci. 2009, 36, 5-17. [CrossRef]

49. Dong, W.; Liao, H.; Roth, R.E.; Wang, S. Eye tracking to explore the potential of enhanced imagery basemaps in web mapping. Cartogr. J. 2014, 51, 313-329. [CrossRef]

50. Manson, S.M.; Kne, L.; Dyke, K.R.; Shannon, J.; Eria, S. Using Eye-tracking and Mouse Metrics to Test Usability of Web Mapping Navigation. Cartogr. Geogr. Inf. Sci. 2012, 39, 48-60. [CrossRef]

51. Holmqvist, K.; Nyström, M.; Andersson, R.; Dewhurst, R.; Jarodzka, H.; Van de Weijer, J. Eye Tracking: A Comprehensive Guide to Methods and Measures; OUP: Oxford, UK, 2011.

52. Brodersen, L.; Andersen, H.H.; Weber, S. Applying Eye-Movement Tracking for the Study of Map Perception and Map Design; National Survey and Cadastre: Norresundby, Denmark, 2002.

53. Castner, H.W.; Eastman, R.J. Eye-movement parameters and perceived map complexity-I. Am. Cartogr. 1984, 11, 107-117. [CrossRef]

54. Oliveira, D.; Machín, L.; Deliza, R.; Rosenthal, A.; Walter, E.H.; Giménez, A.; Ares, G. Consumers' attention to functional food labels: Insights from eye-tracking and change detection in a case study with probiotic milk. LWT Food Sci. Technol. 2016, 68, 160-167. [CrossRef]

55. Hesselmann, G. Applying Linear Mixed Effects Models (LMMs) in Within-Participant Designs with Subjective Trial-Based Assessments of Awareness-a Caveat. Front. Psychol. 2018, 9, 788. [CrossRef]

56. Sameer, A.; Bhushan, B. Effect of Landmark Type on Route Memory in Unfamiliar Homogenous Environment. Psychol. Stud. 2017, 62, 152-159. [CrossRef]

57. Liao, H.; Dong, W. An Exploratory Study Investigating Gender Effects on Using 3D Maps for Spatial Orientation in Wayfinding. ISPRS Int. J. Geo-Inf. 2017, 6, 60. [CrossRef]

58. Hirtle, S.; Richter, K.F.; Srinivas, S.; Firth, R. This is the tricky part: When directions become difficult. J. Spat. Inf. Sci. 2010, 1, 53-73.

59. Kitchin, R.; Blades, M. The Cognition of Geographic Space; I.B.Tauris: London, UK, 2002; p. 12.

(C) 2020 by the authors. Licensee MDPI, Basel, Switzerland. This article is an open access article distributed under the terms and conditions of the Creative Commons Attribution (CC BY) license (http://creativecommons.org/licenses/by/4.0/). 